\title{
Real Time Design and Development of Brain Computer Interface for Home Appliance Using LabVIEW
}

\author{
Kalpak Awale ${ }^{1}$, Anagha Choudhari ${ }^{2}$, Shyam Prasad ${ }^{3}$ \\ PG Scholar, Electronics and Telecommunication Engineering, YCCE, Nagpur, India ${ }^{1}$ \\ Professor,Electronics and Telecommunication Engineering,YCCE, Nagpur, India ${ }^{2}$ \\ Engineer,Tecxtra Technologies Pvt. Ltd, Nagpur, India ${ }^{3}$
}

\begin{abstract}
Each and every physical action we make is provoked by the neural course of action in human brain. With the correct apparatus and contemporary advancement or improvement in both the fields of cognitive neuroscience and brain imaging mechanism. This has drive to tremendous and accelerated growing field of brain computer interface (BCI). The BCI benefits or supports physically disabled, aged or unblessed people to make the use of the devices and application through their mental or cerebral exercise. Remarkably people conclude that $\mathrm{BCI}$ is a boon for physically challenged persons anguish from serious neuromuscular disorders. So in this paper, we evolve a BCI technology that control the home appliance for physically disabled or aged people. The EEG study conception has been applied to control the home appliance such as light or fan automatically that is without doing any physical movement from the place to electrical switch board for immobile persons. The EEG signals are catched from the subject brain activity using "NEUROSKY MINDWAVE MOBILE" EEG sensor which is implanted at the FP1 location of the subject forehead. The depacking and processing of the EEG signals and eye blink data from the raw EEG brain signals is done by using LabVIEW software from which composed signal is generated to control the home appliance. Micro-controller is used to control the relay drive circuitry to handle the on/off switching of the home appliance.
\end{abstract}

Keywords: LabVIEW (Laboratory Virtual Instrument Engineering Workbench), Electroencephalography (EEG),Brain computer interface (BCI),Eye Blinks, Neurosky Mindwave Mobile, Micro-controller.

\section{INTRODUCTION}

A BCI is a control and communication system that does not lean in any means on the normal neuromuscular brain output channels, that is the subject intention is dispatch by EEG brain signals rather than by the peripheral muscles and nerves and these brain signals do not bank on for their formation on neuromuscular activity. As a control and communication system, brain computer interface constitute a real time communication among the subject and the outside world. Electroencephalography is a technique which is used for the evaluation of an electrical activity or signal generated by the human brain.

The human brain is an excessively essential structure or organ in the human body over which a subject is capable to acknowledge to numerous sensory stimuli as well as execute complicated activities. Various exercise such as emotions, language, wakefulness, sleep, cognition and memory are contrived achievable by the human brain. There many facet of the human brain that is still not unplumbed. The three fundamental elements of the brain consists of the cerebrum, brainstem, and cerebellum. The brain stem is responsible for the regulation of an important physiological activity such as heart rate, breathing, and blood pressure. The cerebellum accepts the sensory input from the coordinates muscular movement and the cerebrum. The cerebrum is a vital element of the brain that manages perception, learning, emotion and intelligence. The extensive lobes in the human brain includes temporal lobe, frontal lobe, parietal lobe and the occipital lobe.

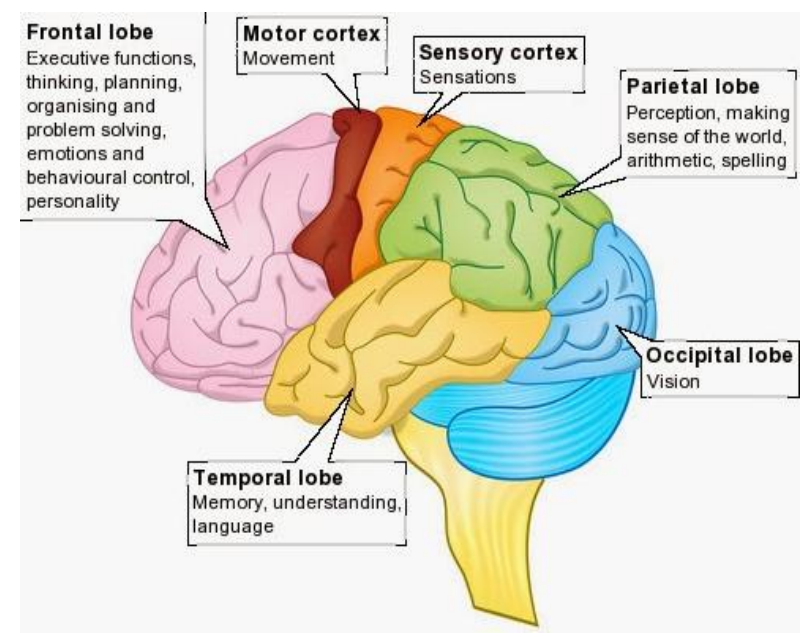

Fig. 1 Basic Structure of Human Brain

A neuron is cell in the body that is specialized to carry messages. The process by which a neurons carries 
messages from one part of the brain to another is through what is called electrochemical process. The electro part happens in the neuron itself. The chemical part happens at function between two neurons called the synapse. There are about 100 billion neurons in the human brain. These neurons have many shapes and come in many sizes. Their job is to allow you to think and behave. An axons carries information away from the cell body to the dendrites of a neighbouring neuron or to another body structure such as a muscle. Axons have smooth surfaces and no spines like dendrites have. Most neurons have only one axon, and the axon branches further from the body that the dendrites does. Myelin sheath is an insulating membrane found around the axons of some neurons.

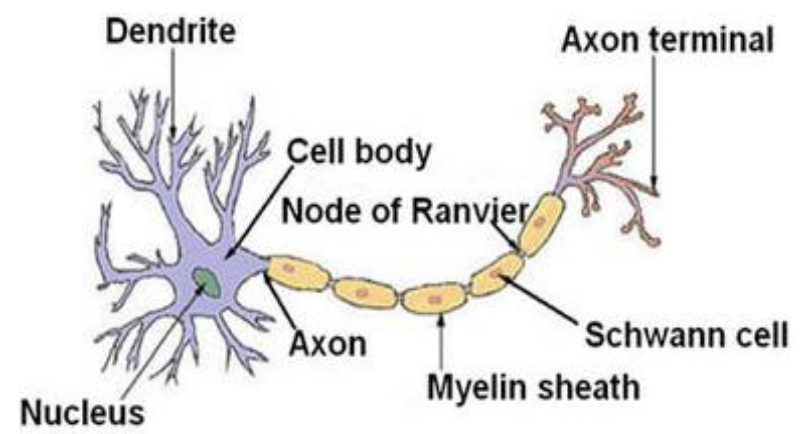

Fig 2 Single Neuron Structure

\section{TYPES OF BCI}

Brain computer interface commenced with the scientist "Han Berger's" invention of electrical activity taking place in the human brain and the advancement of electroencephalography (EEG). For the first time the EEG signals from the human brain was recorded by "Berger" in the year 1924. By the evaluation of the electroencephalography signals from the human brain "Berger" was able to determine the oscillation activity such as alpha waves further recognized as 'Berger' waves. BCI is also known as or probably called as Mind-Machine Interface (MMI) OR Brain-Machine Interface (BMI) is a straightforward communication path between the external world and the human brain.

The various category of BCI that are disclosed are as follows:

1. Invasive Brain Computer Interface

2. Partially Invasive Brain Computer Interface

3. Non Invasive Brain Computer Interface

INVASIVE BCI: Invasive BCI are the type which are inculcated precisely inside the human brain and has the highest signal quality. As they are placed in the grey matter of the human brain, the subject is very much susceptible to build up scar-tissue in the brain leading to weaker signal reception or even get vanished as the human body respond to a foreign substance in the brain. The trouble is that the subject need to undergo a surgery for the implantation of the sensor or the electrode in the brain.

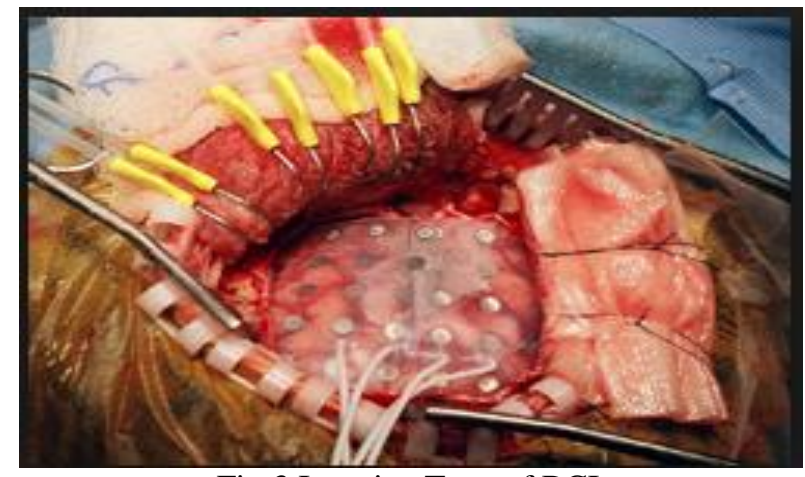

Fig 3 Invasive Type of $\mathrm{BCI}$

PARTIALLY INVASIVE BCI: Partially invasive BCI are the type which are inculcated precisely inside the skull but are placed outside the grey matter as compared to invasive BCI. Signal reception is bit weaker as correlated with invasive BCI. But the probability of the scar-tissue formation in the human brain is very much less. Here also the subject has to undergo a surgery for the implantation of the sensor or electrode.

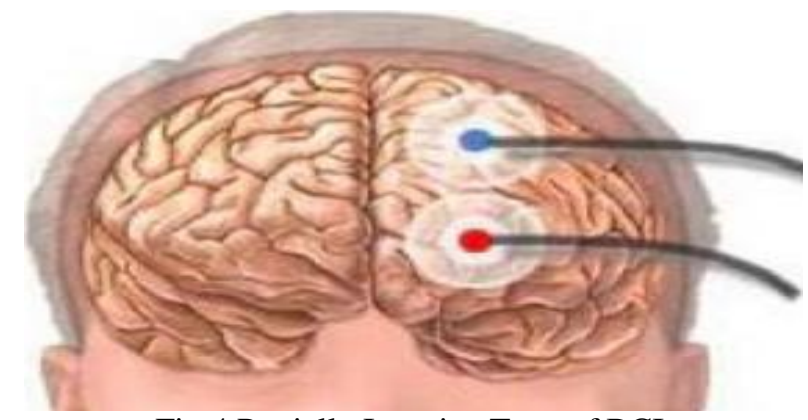

Fig 4 Partially Invasive Type of BCI

NON INVASIVE BCI: In non-invasive BCI type the sensor is directly paced on the surface of the head outside the skull of the human brain, with no need for the subject to undergo surgery as compared to that in invasive and partially invasive type of BCI. There is absolutely no possibilities of having scar tissue or tissue damage in the human brain, hence treated to be the secured technique rather than other two types of BCI. Reception of the signal clarity become little less as the sensor or electrode is not directly placed on the desired or require part of the human brain. The non-invasive BCI type are very popular, easy to use, cheap and even portable.

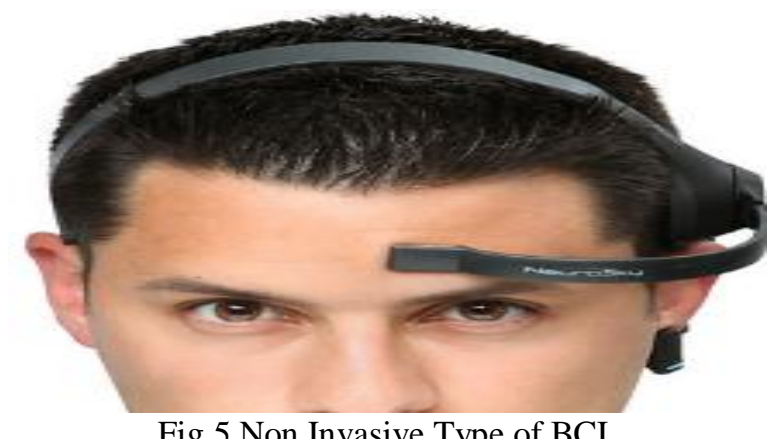

Fig 5 Non Invasive Type of BCI 


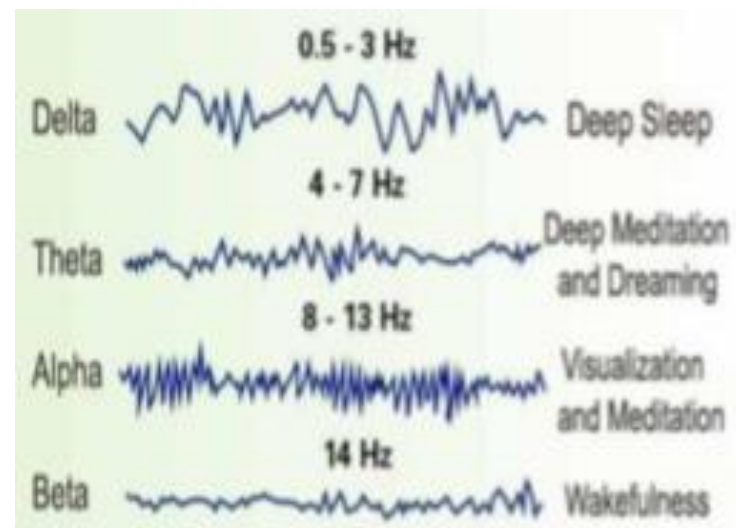

Fig 6 EEG Signals Allocation

\section{III.METHODOLOGY}

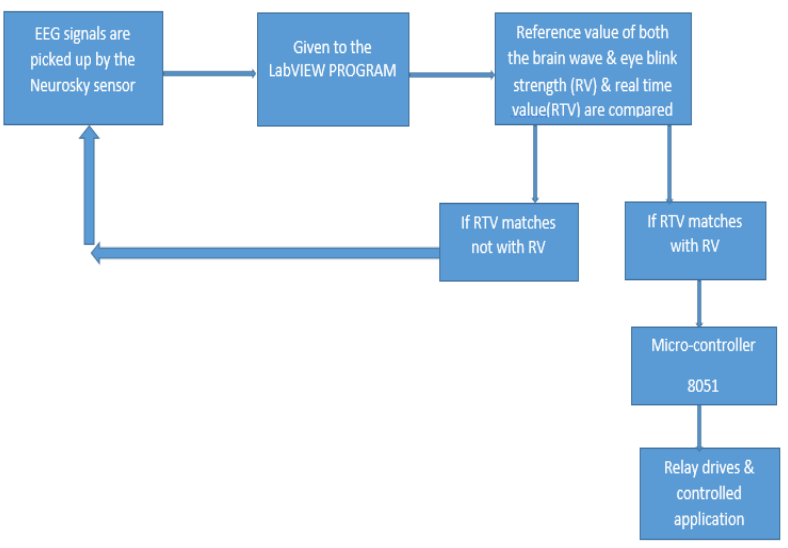

Fig 7 Block Diagram

\section{A. BCI SYSTEM}

Brain computer interface technology is symbolize by the neurosky mindwave mobile headset. Non-invasive type of $\mathrm{BCI}$ is used where the electrode or the sensor is placed on the FP1 location on the forehead which captures the raw EEG data and transmits vice Bluetooth to the control and decision unit that is LabVIEW.
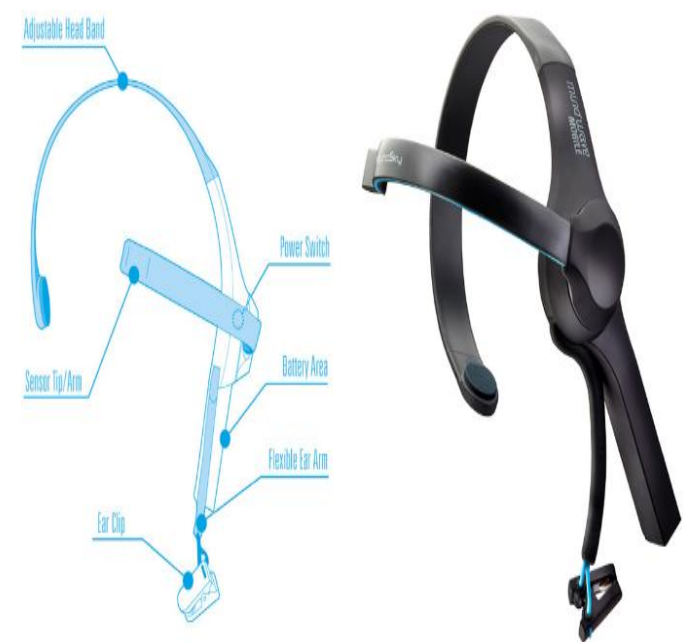

Fig 8 Neurosky Mindwave Mobile Headset
The advantage of the neurosky sensor in comparison with all the other sensors which are there in the market is that it's consist of only two dry single electrodes without the need for applying any conductive gel, for implanting the sensor on the forehead skin. The neurosky mindwave mobile headset consists of a sensor arm and an ear clip. The sensor reference and ground electrodes are on the ear clip sensor and the EEG sensor is on the sensor arm resting on the forehead above the eye (FP1 position).

The physical parameter are as follows:

1. Weight : $90 \mathrm{~g}$

2. Sensor arm up : height : $225 \mathrm{~mm} *$ width : $255 \mathrm{~mm} *$ depth : $92 \mathrm{~mm}$

3. Sensor arm down : height : $225 \mathrm{~mm} *$ width : $155 \mathrm{~mm} *$ depth : $165 \mathrm{~mm}$

4. Single AAA battery.

5. 8 hours battery runtime.

\section{B. BCI COMMUNICATION}

Neurosky sensor present capture and filter out the EEG signal. The electrical pulse generated in the brain is captured by the sensor placed at the FP1 location simultaneously the distortion or noise is drained out by the second sensor that is placed on the ear. Noise is basically generated because of the human movement, muscle movement and other electrical devices. The neurosky mindwave mobile measures following parameter that is raw EEG signal, attention and meditation levels, and EEG signal band that is delta, theta, low alpha, high alpha, low beta, low beta, high beta, low gamma, mid gamma at the sampling rate of $512 \mathrm{~Hz}$ and the baud rate is $9600 \mathrm{bits} / \mathrm{sec}$. the sensor also detects or measure the blinking of the human eye as the sensor is very close in proximity to the eye muscle as it is placed just above the eye at the forehead (FP1 location). The neurosky sensor transmits the data in the form of the packet and communicate via Bluetooth protocol with LabVIEW software by establishing the Virtual Communicationthat is COMM PORT. The headset consists of the Think Gear Communication Module or Driver which calculates all the parameter and transmits out in the form of the packets which in turn are parsed out using proper parsing algorithm or logic in the LabVIEW software through which the control signal is generated which is given to the micro-controller circuitry unit to control the home appliance or application.

\section{SOFTWARE- APPLICATION INTERFACE}

LabVIEW (Laboratory Virtual Instrument Engineering Workbench) is graphical programming language which generates and creates an innovative platform for simple enhancing of a complex features along with error handling application as well as facility of providing correlative interface to the end user. The graphical programming block designing in LabVIEW is called as "Virtual Instrument". The Virtual Instrument consists of two main block that is Front Panel Blockand the Block Diagram Block. The front panel block provide a collaborative API 
to the end user and the block diagram block consists of graphical block arranges in the proper logical algorithm to generate the desired output. The advantage of the LabVIEW is that the labview programming follows dataflow execution model as compared to that in ' $\mathrm{C}$ ' programming which follows control flow execution model. There are total of eleven "Sub VI" in LabVIEW are created for step by step parsing of packets transmitted by the neurosky mindwave mobile headset.

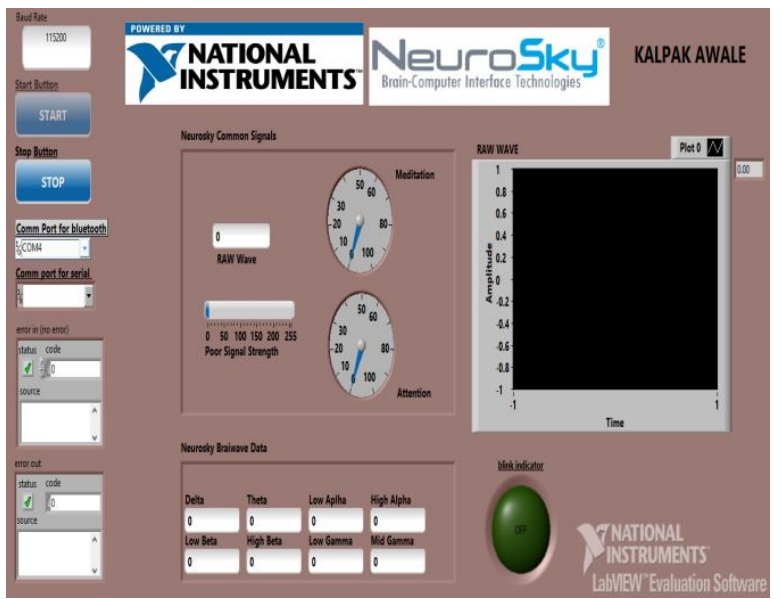

Fig 9 LabVIEW Front Panel

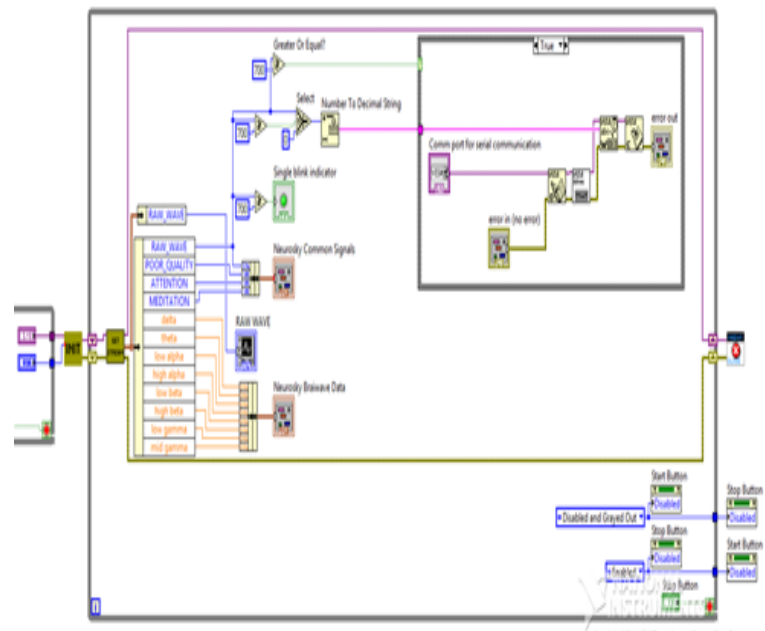

Fig 10 Labview Block Diagram

\section{EYE BLINK DETECTION, MEDIATATION ATTENTION LEVEL MAPPING}

As the sensor is placed just above the human eye on the forehead the movement of the muscle in the ophthalmic part of the human body is easily detected by the sensor as a result it produce a larger amplitude of an impulse in the real time procurement of the raw EEG signal. Hence eye blink detection is possible using neurosky mindwave mobile headset. Practically it was found that spontaneous blink of a human eye result into a shift in magnitude level in between 650 to 700 amplitude. Thus blink of a human eye create a spike above 650 to 700 amplitude in raw EEG signal which is further used as a control signal to do serial \& communication with the micro-controller unit for switching on/off of the home appliance application. The lower threshold value for the eye blink is set to 700 to generate a control signal depending upon the intensity of the eye blink of an end user. In addition to the eye blink detection the raw EEG signal also consists of attention and meditation level which is in the scale of 0 to 100 as shown in the front panel. The attention level relates that whether the user is more attentive or focused, as the reading is above 80 that mean the user is more attentive and less than 20 the user is less attentive and in between mean the user is moderate attentive and similar for the meditation level. The meditation level relates that how relaxed and calmed is the user corresponding to the meditation level readings. The poor signal strength on the front panel indicates the quality of the received raw EEG signal from the sensor which is in the range of 0 to 255 . Zero indicates the quality of the signal is very good and 200 and above indicates that the sensor is not touched to the forehead skin resulting into bad quality of the EEG signal.

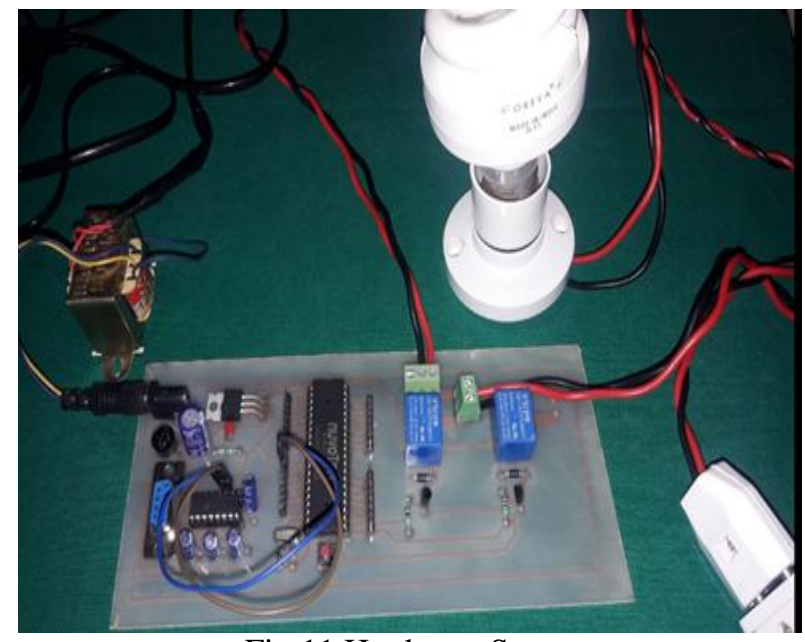

Fig 11 Hardware Setup

\section{E. HARDWARE SETUP}

The hardware setup consists of following components:

1. 230V step down transformer.

2. Bridge rectifier, LED, voltage regulator IC 7805 .

3. IC MAX 232, Micro-controller 8051

4. USB to SERIAL convertor, BC 557 PNP.

5. Relay, diode, light bulb, resistors and capacitors.

IC MAX 232 is a 16 pin integrated circuit most commonly used in voltage level signal problem. Basically IC is used in RS 232 communication system for conversion of voltage level on TTL device that are integrated with the PC port and the micro-controller. The IC work as a hardware layer convertor to communicate between two systems simultaneously.

\section{F. FUTURE SCOPE}

The future scope is that further other home appliances can also be controlled using double eye blink and triple eye blink detection. 


\section{IV.CONCLUSION}

The technology of Brain Computer Interface is more demanding to the modern science. The possibility of BCI open the advanced channel of interaction in the field of medicine, psychology, military and media. BCI opens a new channel of communication for the disabled or medical disorder or aged people who gradually lose their control on their own body and reach to state where they have no potential to communicate with the external environment. Hence BCI creates an innovative platform for all these group of people to interact with external environment in the similar fashion as the normal human being to some extent. Hence the control of home appliance such as light bulb switching on/off with eye blink of a human eye using neurosky mindwave mobile headset (BCI technology) is concluded.

\section{REFERENCES}

[1] C.W. Anderson and Z. Sijercic. Classification of EEG signals from four subjects during five mental tasks. Inrl. Con\$ on Engineering Applications of Neural Networks, 407414, 1996.

[2] Lal, T.N, Schr"oder, M.T, Hinterberger, J., Weston, M., Bogdan, N., Birbaumer, B and Sch"olkopf, "Support Vector Channel Selection in BCI", in the proceedings of IEEE Transactions on Biomedical Engineering, Special Issue on Brain-Computer Interfaces, June 2004.

[3] Anupama.H.S, N.K.Cauvery, Lingaraju.G.M, "Brain computer interface and its types - a study", International Journal of Advances in Engineering \& Technology, May 2012.

[4] L. Mayaud, M. Congedo, A. van Laghenhove et aI., "A comparison of recording modalities of P300 Event Related Potentials (ERP) for Brain-Computer Interface (BCI) paradigm, " Clinical Neurophysiology, vol. 43, no. 4, pp. 217-227, 2013.

[5] H. Bo, "EEG analysis based on time domain properties, " Electroencephalography and Clinical Neurophysiology, vol. 29, no. 3, pp. 306-310, 1970.

[6] Lecuyer, A., Lotte, F., et al. "Brain-Computer Interfaces, Virtual Reality, and Videogames", IEEE Computer, IEEE Computer Society, 41(10): 66-72, 2008.

[7] Vidal, J.J. "Toward direct brain-computer communication", Annual review of biophysics and bioengineering, Vol. 2, 157-80, 1973.

[8] J. R. Wolpaw and D. J. McFarland, "Control of a two-dimensional movement signal by a noninvasive brain-computer interface in humans," PNAS, pp. 0403504101, 2004.

[9] G. Schalk, D. J. McFarland, T. Hinterberger, N. Birbaumer, and J. R. Wolpaw, "BCI2000: a general-purpose brain-computer interface (BCI) system," IEEE Transactions on Biomedical Engineering, vol. 51, pp. 1034-43, 2004.

\section{BIOGRAPHIES}

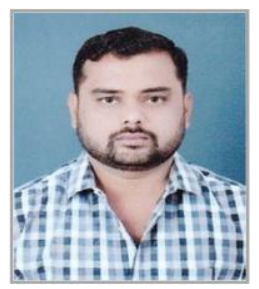

Kalpak Awale is currently pursuing his Master of Technology (MTech) program in Communication Engg from Yeshwantrao Chavan College of Engineering, Nagpur, India. He has completed his Bachelor of Engineering (B.E) program in Electronics and Telecommunication Engg from Mumbai University.

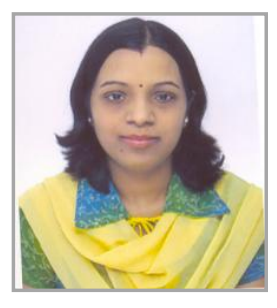

Mrs. Anagha V Choudhari, MTech in Electronics Engineering from RTM Nagpur University, is working as Assistant Professor in the Department of Electronics and Telecommunication Engineering, Yeshwantrao Chavan College of Engineering, Nagpur, Maharashtra, India. She has a total teaching experience of 13 years. Her area of interest is Embedded Systems.

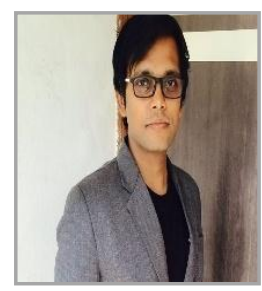

Mr Shyam Prasad, Softronic Engg, is currently working in Tecxtra Technologies Pvt. Ltd. He has a total 6 years of Industrial experience. 